\title{
Der Palmengarten im Jahr 2014
}

\author{
Matthias Jenny \& Manfred Wessel
}

\section{Januar}

Draußen ist es zwar grau und trüb, und es stellt sich kein Winter mit Eis und Schnee ein. Dennoch glitzern bereits seit Dezember des vergangenen Jahres die Winterlichter und verzaubern den Palmengarten in eine märchenhafte Welt: Elfen tanzen in den Bäumen, Diskolicht-Glühwürmchen blinken im Bambushain, und Fahrräder schweben über der Steppenwiese. In der Galerie Ost können es sich Besucherinnen und Besucher mit heißen Getränken und einem kleinen Imbiss gemütlich machen sowie im Märchenzelt spannenden Geschichten lauschen.

Auch wenn es eigentlich noch Winter ist, kündigt sich der Frühling bereits an. Es steht nun fest, dass es zum Frühlingsbeginn einen Frühlingsball im Festsaal geben wird. Damit wird die alte Tradition, rauschende Feste und Bälle im Gesellschaftshaus zu feiern, nach langer Zeit wieder zum Leben erweckt. Alexandra Prinzessin von Hannover, Petra Roth und Renate von Metzler machen sich für diesen Ball stark. Die Stiftung Palmengarten und Botanischer Garten als Veranstalterin des neuen Frühlingsballes möchte mit dem Erlös die beiden Traditionseinrichtungen nachhaltig fördern. Der diesjährige Ball steht unter dem Zeichen „Blüten und Schmetterlinge“, um das für die nahe Zukunft geplante Blüten- und Schmetterlingshaus finanziell zu unterstützen.

Aufgrund der milden Temperaturen kann auch im Garten bereits ab Januar "gebuddelt" und mit den Arbeiten an der neuen Fernwärmetrasse begonnen werden.

Leider muss eine fast 100-jährige WinterLinde neben dem Café Siesmayer gefällt werden, da sie von einem Pilz befallen und nicht mehr standsicher ist. Eine Quercus alba wird daneben nachgepflanzt.

Mit Ende des ersten Monats des Jahres erlöschen die Winterlichter, die von 18000 Besuchern bewundert wurden.

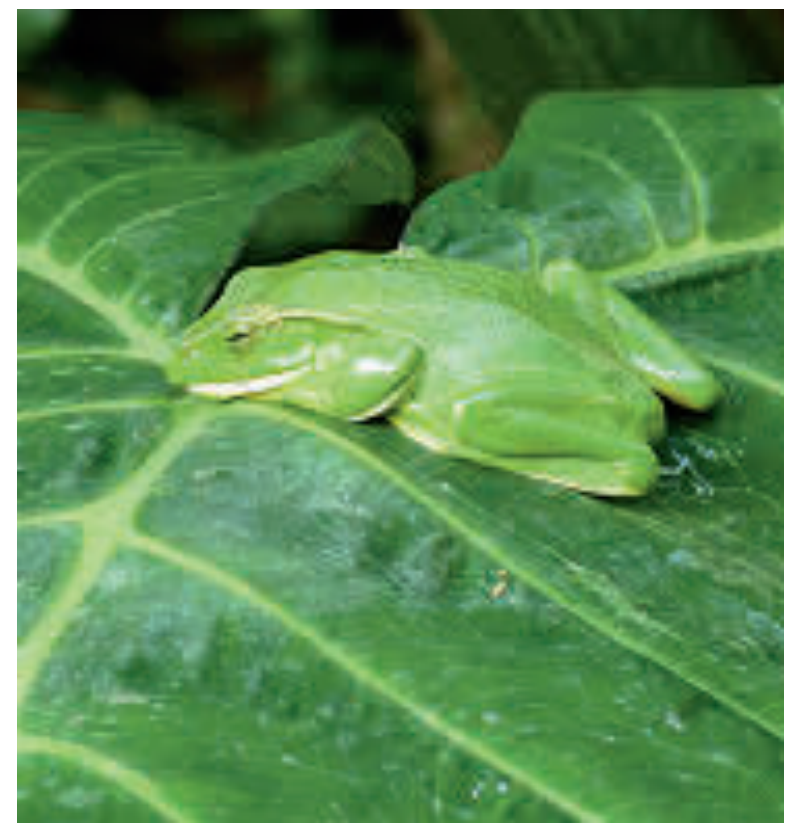

Damit der Palmengarten seine Gäste besser informieren kann, gleichzeitig aber auch eine Gartenaufsicht präsent ist, wird ein Besucherservice eingerichtet.

Im Botanischen Garten findet Mitte Januar der schon traditionelle Winterspaziergang statt. Bei der Führung können die rund 50 Besucher einige frühe Blüher bewundern, z.B. die Stinkende Nieswurz (Helleborus foetidus), die am sonnigen Kalkhang besonders auftrumpft, da sie ihren Pflanzenkollegen mal wieder weit voraus ist.

Schon richtig frühlingshaft bunt mit blühenden Krokussen auf den Wiesen geht es in den Februar. Kein Wunder, denn die Januartemperaturen lagen vier Grad über dem Durchschnitt. Ein spannendes Jahresprogram mit Führungen und Vorträgen wurde bereits seit Jahresbeginn wieder gut angenommen.

Abb. 1: Wenn es im Winter draußen kalt ist, lohnt sich ein Spaziergang durch die Gewächshäuser. Neben vielen Pflanzen kann man dort auch einzelne Tiere beobachten wie z. B. im Tropicarium den Fea's Riesenruderfrosch (Rhacophorus feae). 


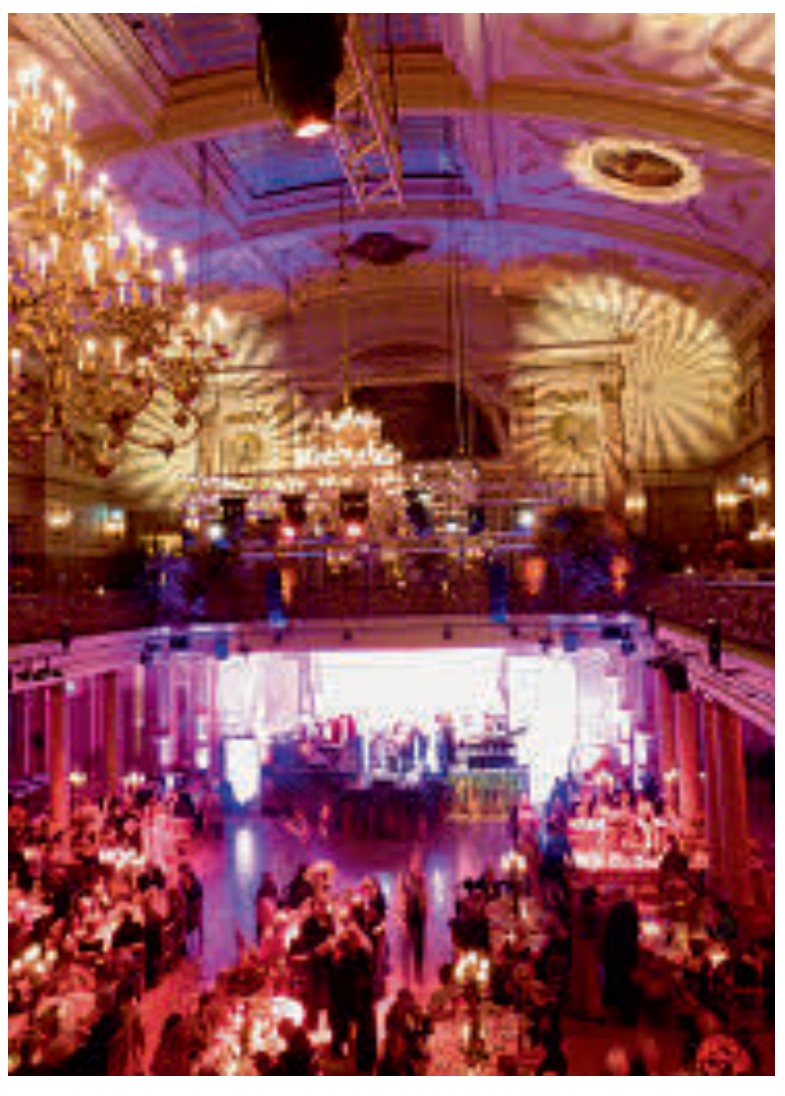

Ein Genuss für Augen und Nase ist jedes Jahr die Frühlingsblumenausstellung in der Galerie, wo Zehntausende Frühlingsblumen ihre Blüten entfaltet haben und den Raum mit ihrem süßen Frühlingsduft füllen. Auch im Blütenhaus ist es Frühling geworden. In dem relativ kleinen Schauhaus kommt der Blütenduft besonders intensiv zur Geltung.

Mit dem Ende der Frühlingsblumenausstellung fällt am 1. März der Frühlingsball zusammen. 10000 Blumen schmücken den Festsaal. Die Gäste sind begeistert, es wird edel diniert, danach flaniert und getanzt. Für musikalische Unterhaltung sorgt der Sänger Thomas Anders. In der Presse wird der Frühlingsball liebevoll als "Tanz für die Schmetterlinge“ bezeichnet. Es steht danach fest, dass es auch in den kommenden Jahren einen Frühlingsball geben wird.

Nicht nur während der Ballnächte kann im Palmenaus genussvoll unter Palmen gewandelt werden. Da es für die Koikarpfen im Becken unter dem Wasserfall dabei manchmal zu unruhig wird und gelegentlich Dinge im Wasser landen, die für Fische gefährlich werden können, bekommen die Kois ein neues Zuhause.

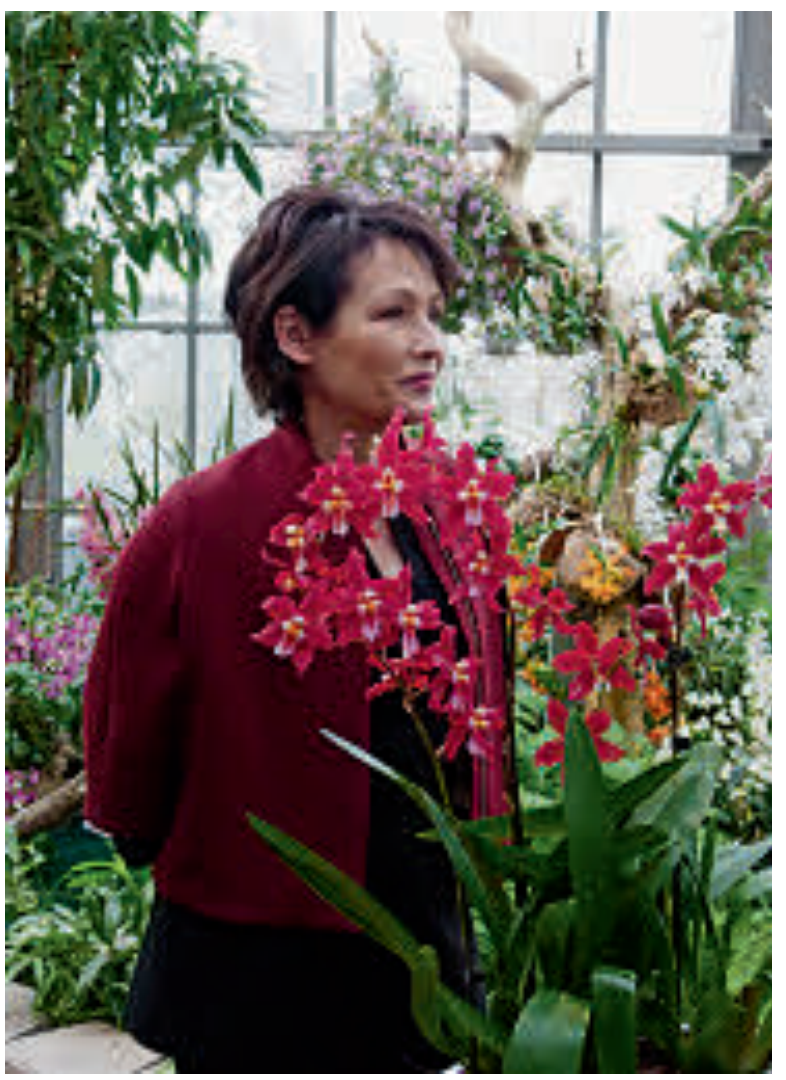

Sie werden in das Becken im Nebelwaldhaus im Tropicarium umgesiedelt.

Bei schönem Frühlingswetter bekommt vermutlich jeder Lust auf Garten. Die traditionelle Gartenmesse ist umkonzipiert, heißt nun Grüne Börse und findet vom 21.-23.3. statt. Schwerpunkt sind Informationen rund um den grünen Bereich sowie die Raritätenbörse. Zeitgleich läuft die Orchideenschau, sodass es für alle Pflanzenfreunde ein vielfältiges Angebot gibt.

Ende Februar wird die Saison im Botanischen Garten offiziell eröffnet. Bei frühlingshaften Temperaturen ist der Garten sehr gut besucht und auch die angebotene Führung lockt wieder über 70 Menschen an, die dem Technischen Leiter durch den Garten folgen. Der Freundeskreis hat diese Veranstaltung in

Abb. 2 (oben links): Frühlingsball im Festsaal.

Abb. 3 (oben rechts): Taufe der Odontioda 'Sevenich' während der Orchideenschau.

Abb. 4 (Seite 5): Grüne Börse. 
bewährter Manier organisiert und sorgt für eine angenehme Atmosphäre.

Ende März sind die Tage schon deutlich länger. Mit Einbruch der Dunkelheit leuchtet es in Frankfurt ab 20:00 Uhr im Rahmen der Luminale an vielen Orten der Stadt. Viele Fotografen sind vom 30. März bis zum 4. April mit guten Kameras und Stativen bis spät in den Abend hinein unterwegs. Ein besonderer Glanzpunkt ist dabei immer der Palmengarten mit mehreren Lichtinstallationen. Diesmal begeistert insbesondere eine brasilianische Installation (Lacrimas de Sao Pedro). Dabei hängen unzählige mit Wasser gefüllte Glühbirnen von der Decke des Hauses Rosenbrunn herunter. Sie wirken wie überdimensionale Regentropfen in einem kräftigen Tropenschauer.

Regelmäßig Anfang April öffnet die alte Yulan-Magnolie vor der Direktion ihre großen weißen Blüten, auch wenn der Baum von einem Pilz befallen ist und jedes Jahr Äste verliert. Diesmal ergibt sich ein etwas anderes Bild, denn die Direktion wurde aus Denkmal- und Gebäudeschutzgründen von seiner alten Fassadenbegrünung aus Efeu und Wildem Wein befreit. In der Azaleenausstellung blühen die Pflanzen sehr üppig, denn das warme Wetter hat gefördert, dass sich alle ihre Blüten schnell geöffnet haben. Die Galerie ist deshalb in ein Blütenmeer in Pink getaucht.

Im April bietet der Botanische Garten seinen Fans erstmals eine Wildkräuter-Führung an. Bei dieser werden essbare Wildkräuter vorgeführt und eine Verkostung angeboten. Eine Vielzahl an Verwendungsmöglichkeiten wird vorgestellt - dieses Angebot wird überdurchschnittlich gut aufgenommen, die Teilnehmerzahl ist riesig und die teilnehmenden Besucher sind sehr angetan.

Von Mai bis in den September hinein ist der Palmengarten ganz „dufte“. Die diesjährige Jahresausstellung widmet sich dem Thema „Duft-Natur und Kultur“.

In den Themengärten des Freilandes sowie auf den Beeten unter Glas gibt es immer wieder Veränderungen. Offiziell eingeweiht ist nun das neu gestaltet Monte-Alto-Beet und eine dazugehörige kleine Ausstellung. Das Beet wurde in

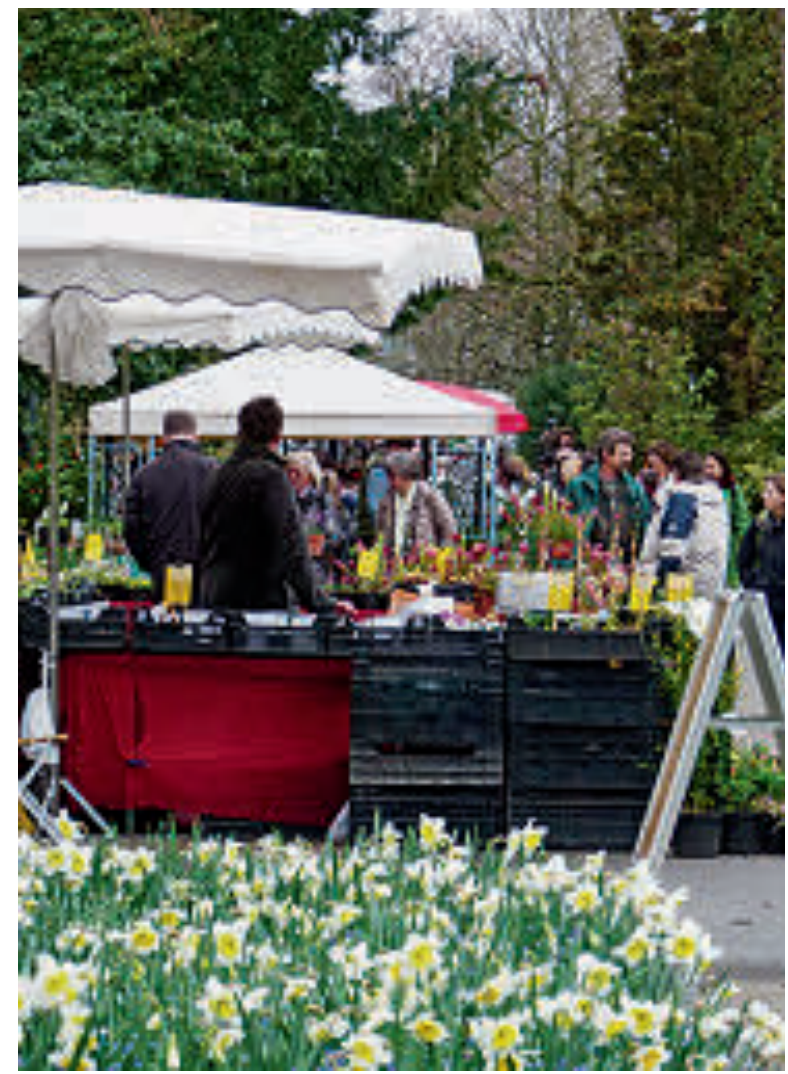

Kooperation mit dem Regenwaldschutzverein Tropica Verde gestaltet. Hingewiesen werden soll dabei auf die Schutzwürdigkeit und Bedrohung des Gebietes sowie auf ein Aufforstungsprojekt in Costa Rica. Im Mai sprießen alle Kräuter, weshalb nun das Grüne-Soße-Festival stattfindet, an dem sich auch der Palmengarten beteiligt.

Begutachtet wird auch die vertikale Begrünung der Lärmschutzwand an der Miquelallee, und es wird entschieden, welches die beste Musterfläche ist.

Die alljährliche Pflanzenbörse im Botanischen Garten findet erstmals nach 17 Jahren an einem neuen Platz statt. Aufgrund der Errichtung einer Kindertagesstätte in Containerform ist der angestammte Platz nun nicht mehr verfügbar. Genutzt wird der Betriebshof, der sich dafür als wunderbar geeignet herausstellt. Die ehemaligen Kolleginnen und Kollegen, die nun im Wissenschaftsgarten der Universität auf dem Campus Riedberg arbeiten, bereichern die Börse mit ihren Pflanzen, sodass dieser Tag wieder ein schöner Erfolg mit vielen zufriedenen Menschen wird. 


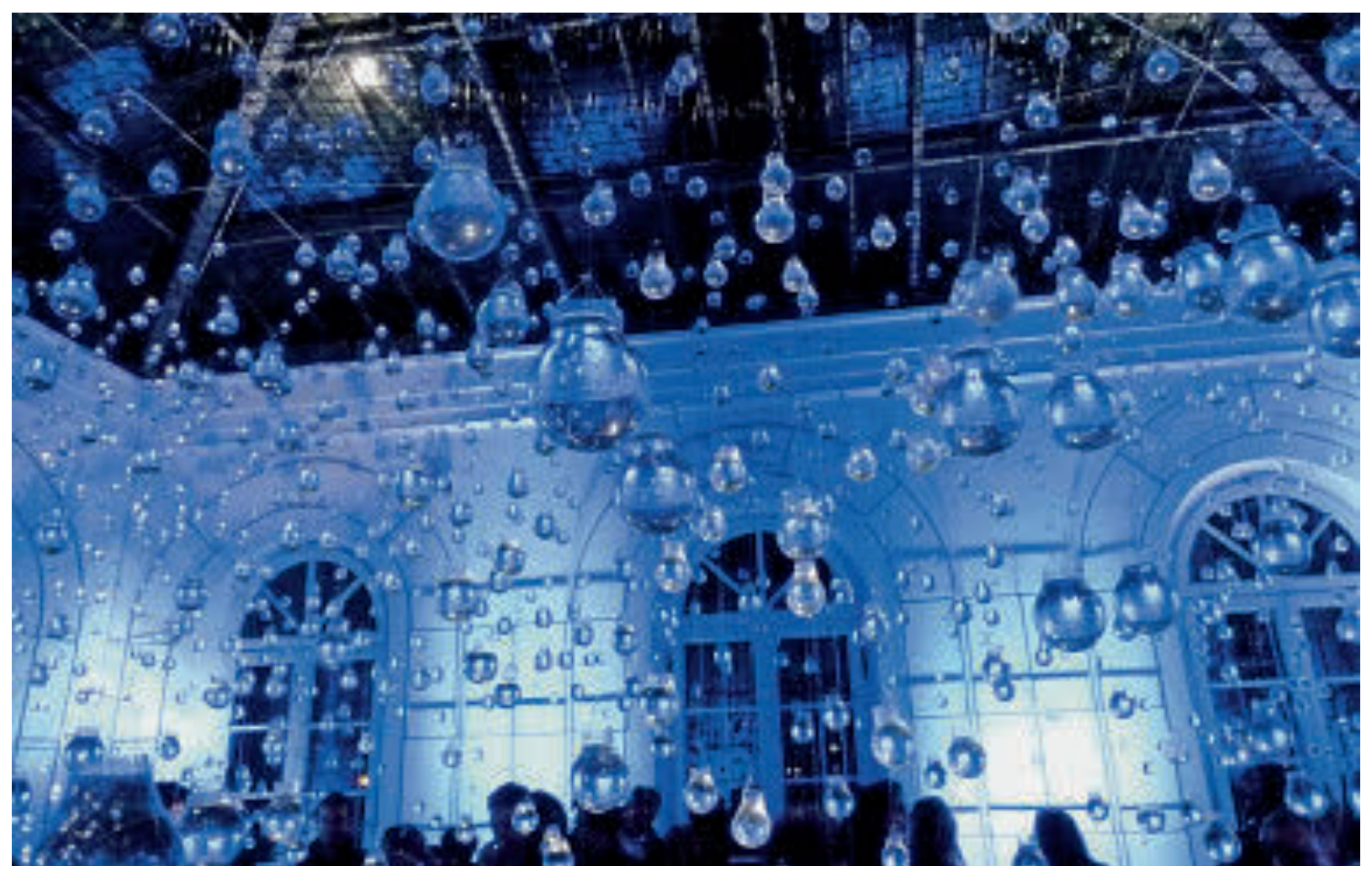

Im Juni dreht sich alles um die Rose. In Jahren mit einem langen, strengen Winter wird oft gebangt, ob die Rosen bis zum Rosenfest richtig schön blühen. In diesem Jahr ist es eher umgekehrt, die milden Temperaturen im Frühjahr haben den Rosengarten bereits ab Ende Mai erblühen lassen. Das Rosen- und Lichterfest findet an einem heißen Bilderbuch-SommerWochenende statt. Es strömen so viele Menschen zu unserem Traditionsfest, dass die Kassen vorzeitig vor dem Feuerwerk geschlossen werden müssen und niemand mehr eingelassen wird, da der Garten fast wörtlich aus allen Nähten platzt. 33000 Besucher genießen an diesem Abend die Königin der Blumen, Picknick auf den Wiesen und die vielen leuchtenden Bilder aus unzähligen Teelichtern. Auch Dezernentin Rosemarie Heilig und andere Stadtprominente bewundern die Rosen während des Dezernatsempfangs, der seit einigen Jahren in der Rosenausstellung stattfindet.

Die Bienen im Botanischen Garten sind jedes Jahr Thema bei zwei Führungen zum Bienenhaus und zu den Wildbienen-Informationskästen sowie der Wildbienen-Brutwand, auch „Bienenhotel“ genannt. Seit vielen Jahren schon gibt es ein Bienenhaus, in dem der Vorsitzende des Freundeskreises des Botanischen Gartens Honig erzeugt, der köstlich schmeckt und auch gekauft werden kann. Der Imker selbst erzählt immer wieder Neues zu seinen Bienenvölkern und der damit verbundenen Arbeit.

Im Juli blüht der Garten üppig, besonders reizvoll ist dann z. B. die Steppenwiese, in der an sonnigen Tagen stets auch viele Schmetterlinge und andere Insekten zu beobachten sind. Das umfangreiche Musikprogramm lockt bis in den September viele Musikfreunde in den Garten. Der Sommer ist auch Zeit der Feste. Das GWH-Familienfest bietet wie in jedem Jahr Groß und Klein einen abwechslungsreichen Nachmittag. Ergänzend zur Duftausstellung kann hier an vielen Pflanzen und Gewürzen geschnuppert werden. Eine mehrjährige Tradition hat nun auch das abendliche Bildzeitungsfest.

Abb. 5 (oben): Luminale mit „Glühbirnen-Tränen“ im Haus Rosenbrunn.

Abb. 6 (Seite 7 oben): Immer wieder ein Hingucker im Tropicarium ist Aristolochia grandiflora.

Abb. 7 (Seite 7 unten): Pflanzenbörse im

Botanischen Garten. 
Im Juli kann man im Botanischen Garten den im Winter 2012/13 neu aufgebauten und gestalteten Basaltbach unterhalb des Alpinums in schon gut entwickelter Pracht bei einer Führung (und natürlich auch sonst) bewundern. Zwar unscheinbar daherkommende kleine Pflänzlein, aber botanisch hochinteressante und vor allem seltene Juwele wie der DreifingerSteinbrech (Saxifraga tridactylites) oder der Zweifelhafte Grannenhafer (Ventenata dubia) sind bereits schön anzuschauen.

Der August gibt sich feucht und kalt. Trotzdem gedeiht der Sommerflor üppig. Die Bepflanzung des Blumenparterres greift das Duftthema auf, indem einige Gewürzkräuter verwendet wurden. Von unseren Giganten blühen im Tropicarium Aristolochia grandiflora, Amorphophallus titanum und Grammatophyllum speciosum. Im Steingarten haben sich die Gunnera-Pflanzen mit ihren riesigen Blättern zu Prachtexemplaren entwickelt. Und der Palmenexpress hat endgültig seine Kinderkrankheiten überwunden und fährt nun wieder regelmäßig.

Im September dominieren die Farben Gelb, Rot und Orange im Garten. Die Erntedankschau ist eine unserer farbenfrohesten Ausstellungen mit Früchten und Gemüse der Saison. Das Herbstfest lockt viele Familien in den Gar-

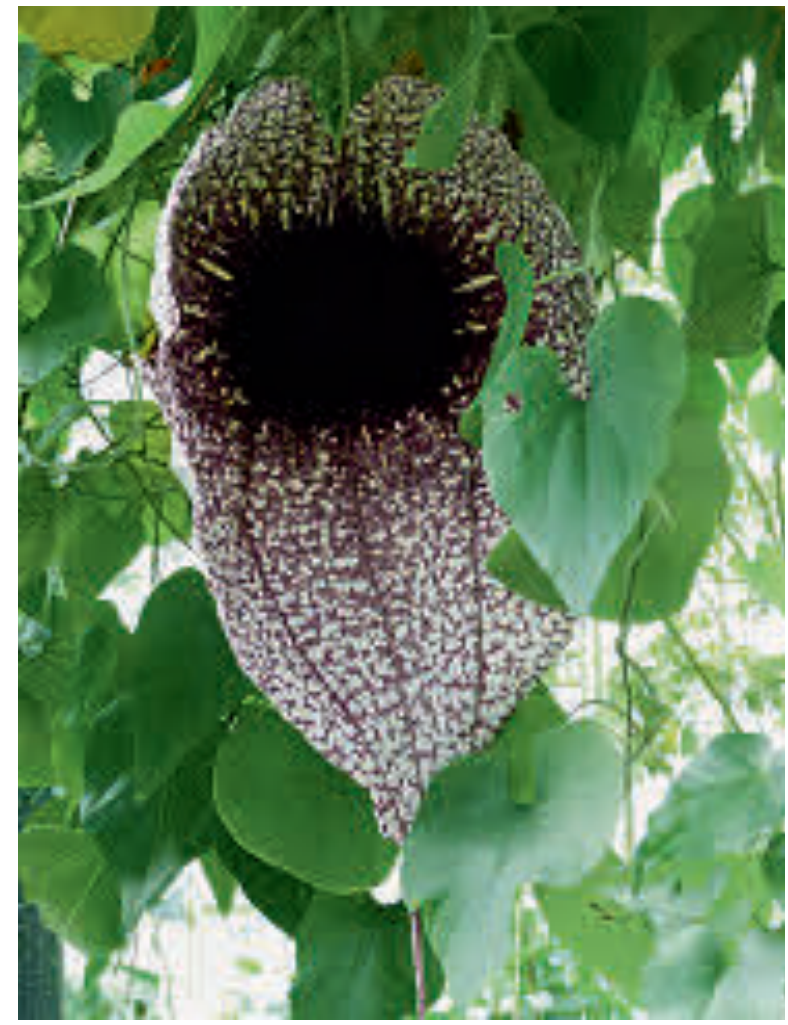

ten. Es können z. B. Nistkästen gezimmert oder Kürbisse geschnitzt werden. Den Abschluss des Herbstfestes bildet ein fröhlicher Laternenumzug durch den Garten.

Der Palmengarten präsentiert sich im Herbst regelmäßig auf dem Fürstlichen Gartenfest auf

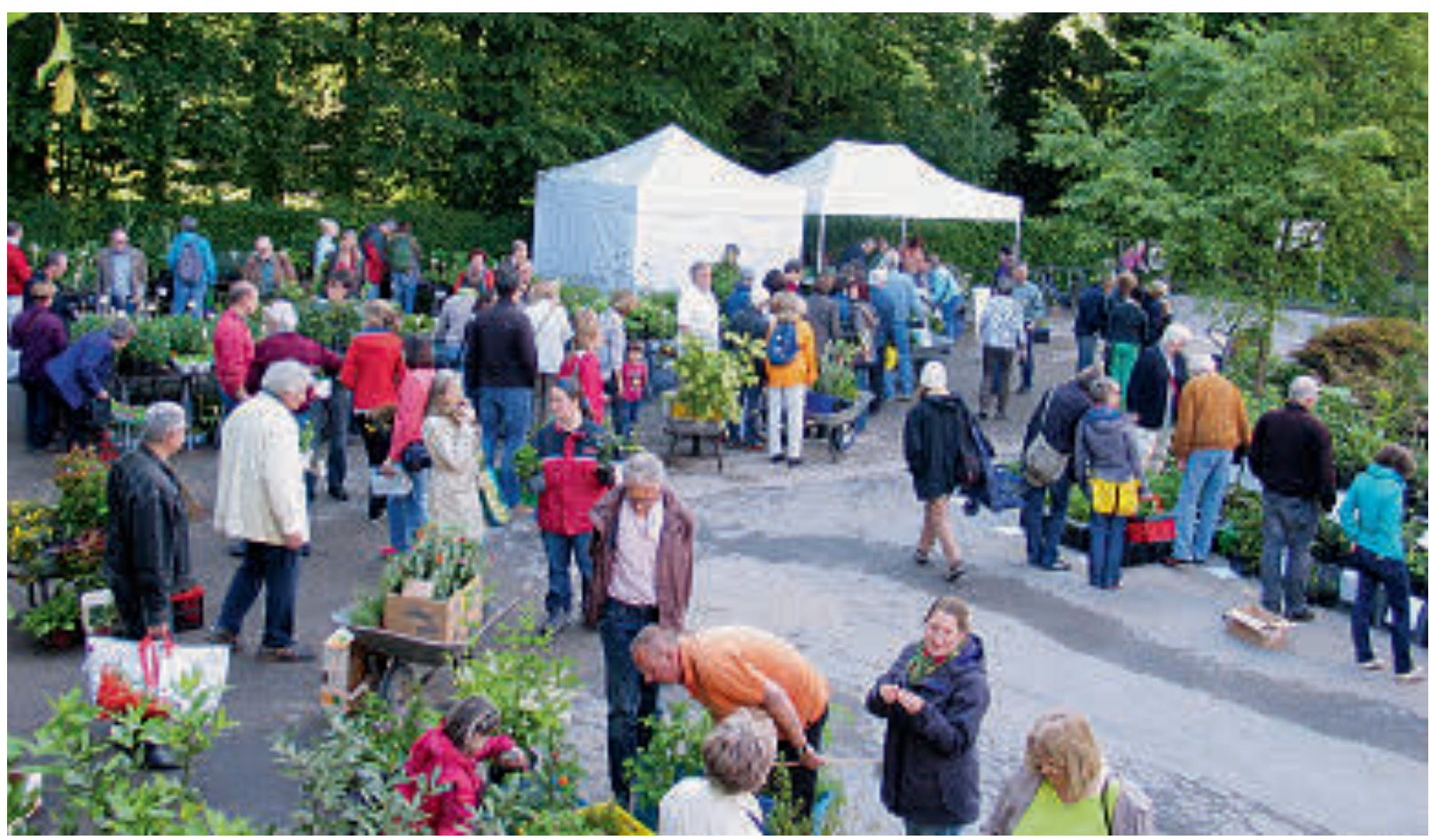




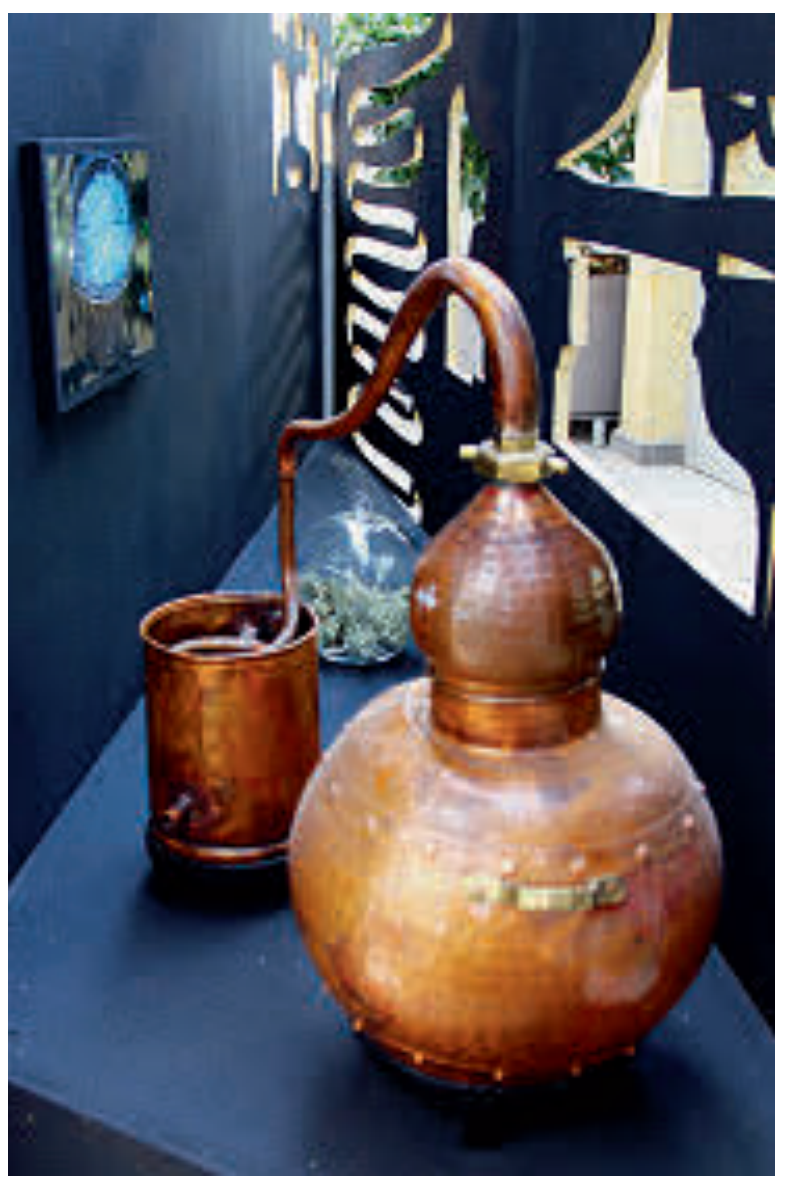

Schloss Wolfsgarten in Langen. Passend zum Thema „Der kulinarische Garten“ ziert ein reich mit essbaren Dingen geschmückter Tisch den Palmengarten-Informationsstand, wofür er mit dem Bronzenen Wolfskopf (Pressepreis) ausgezeichnet wird. Im Freien bringen noch einmal die Dahlien reichlich Farbe in den Garten.

Der 18. Tag der offenen Tür im Botanischen Garten steht an. Wieder kommen zahlreiche Menschen, es gibt ein umfangreiches Angebot an Führungen, Aktionen und anderen Angeboten, und wie immer hören wir mindestens einmal den Satz „Wir leben schon so lange in Frankfurt, aber den Botanischen Garten kannten wir noch gar nicht!" Auch um diesen Missstand $\mathrm{zu}$ beenden, arbeiten die Gärtnerinnen und Gärtner tagein und -aus im Botanischen Garten und engagiert sich der Freundeskreis mit seinen über 450 Mitgliedern sehr intensiv an der Fortentwicklung und Bewahrung dieses Gartens.

Von Oktober bis in den November hinein begeistert botanische Malerei der Society of Bo- tanical Arts mit filigranen Pflanzenabbildungen. Diesmal stehen Gift- und Heilpflanzen auf dem Programm. Die Bilder sind käuflich, und so manches Stück wechselt seinen Besitzer. Wer lieber echte, lebende Pflanzen mag, kann sich während der Orchideen-Verkaufsausstellung mit der Königin der Blumen eindecken.

Eine Umfrage hat ergeben, dass FrankfurtTouristen das Museumsufer, den Palmengarten und das Senckenberg-Museum ganz besonders lieben. Unter den Parks nimmt der Palmengarten Platz Nummer eins ein.

Der 31. Oktober markiert wie gewohnt das Ende der Besuchssaison im Botanischen Garten. Ein erfolgreiches Gartenjahr neigt sich dem Ende entgegen, obwohl: für die Gärtnerinnen und Gärtner ist die Arbeit damit nicht beendet, im Gegenteil. Es werden die vielen notwendigen Jahresabschlussarbeiten geplant und in Angriff genommen, der Teich muss noch einmal gesäubert, das viele Laub aufgesammelt und kompostiert, Gehölze geschnitten, empfindliche Stauden vor der Kälte geschützt werden und vieles mehr. Und das nächste große Projekt, die Sanierung und Versetzung der alten Ostasien-Natursteinmauer entlang des Hauptweges zum Bienenhaus, wird geplant und vorbereitet. Das nächste Gartenjahr steht schon vor der Tür.

Im November bereichern Chrysanthemen unseren Ausstellungstournus. Nun endlich sind auch wieder Heinrich und Elisabeth SiesMAYER vereint. Das Ölbild des Gartengründers hing bisher alleine ohne seine Gattin im Büro des Amtsleiters. Siesmayers Urenkel, Uwe FeHLer, stiftete nun das dazugehörige Bildnis von Elisabeth. Beide Siesmayers hängen nun

Abb. 8 (oben): In der Ausstellung „Duft“.

Abb. 9 (Seite 9 oben links): Die Dahlien vor dem Haus Leonhardsbrunn kündigen den Herbst an.

Abb. 10 (Seite 9 oben rechts): Sommerflor vor dem Eingangsschauhaus.

Abb. 11 (Seite 9 unten links): Herbst im Botanischen Garten.

Abb. 12 (Seite 9 unten rechts): Schachbrettblumen unter den englischen Blumenmalereien. 

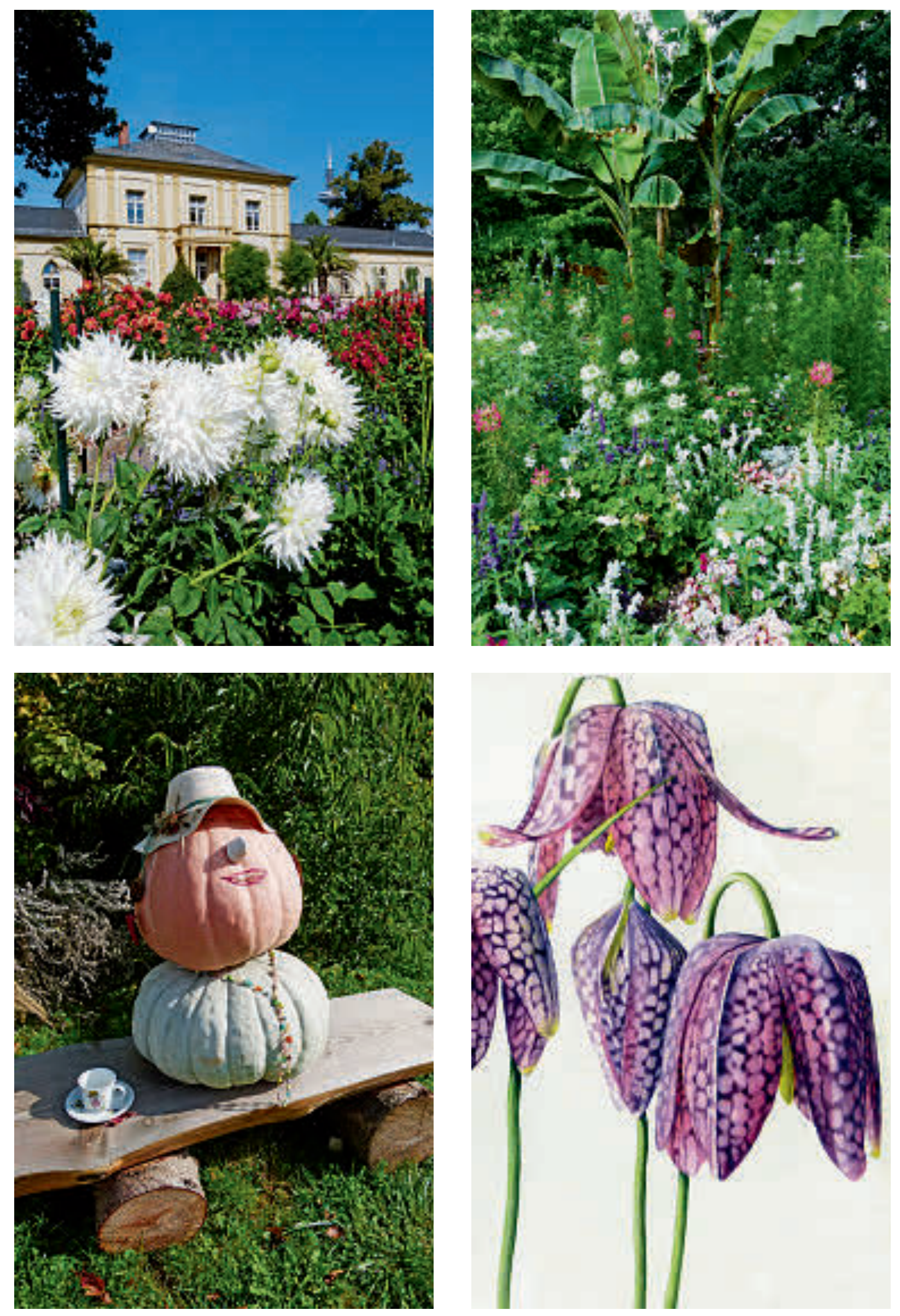


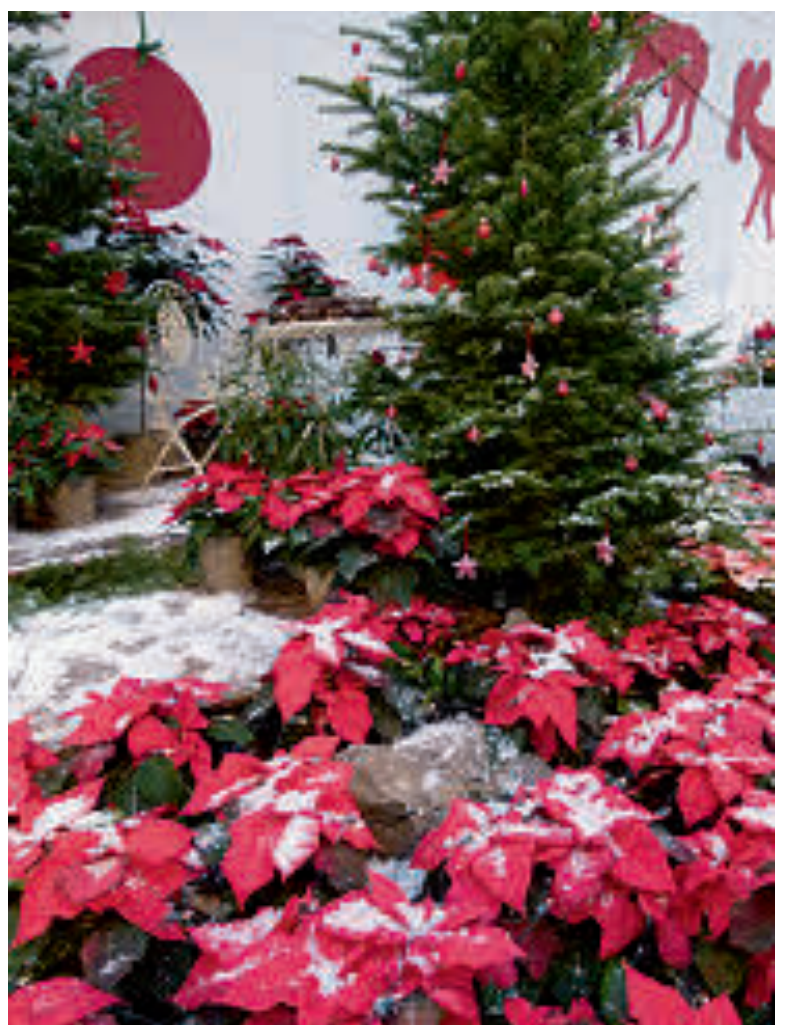

vereint in einem Sitzungszimmer des Palmengartens. Das Blütenhaus hat sich wieder in eine Orangerie verwandelt, in der diverse, aktuell fruchtende Zitruspflanzen gezeigt werden. In dieser Form wird es das letzte Mal sein, denn es steht nun fest, dass mit dem Abriss des alten Blütenhauses und dem Neubau des modernen Blüten- und Schmetterlingshauses im kommenden Jahr begonnen werden kann.

Der Winter will sich nicht so richtig einstellen. In der Weihnachtsausstellung gibt es im Dezember aber Kunstschnee, stimmungsvoll geschmückte Weihnachtbäume, viele Weihnachtssterne oder Amaryllis. Etwas später werden auch wieder die Winterlichter eingeschaltet, diesmal galoppieren bunte Pferde und ein Einhorn über die Wiese, blickt ein großer sitzender und leuchtender Mann auf den Messeturm oder haben sich die leuchtenden Schneeglöckchen im Vergleich zum letzten Mal vermehrt. Das Winterlichter-Café in der Galerie ist ein angenehmer Ort, sich nach dem abendlichen Spaziergang durch den Garten aufzuwärmen. Der Schnee verpasst nur ganz knapp die Weihnachtstage, sodass bis Sylvester die Illumination besonders winterlich wirkt.

Abb. 13 (oben): Weihnachtsausstellung.

Abb. 14 (unten): Während der Winterlichter illuminiertes Direktionsgebäude.

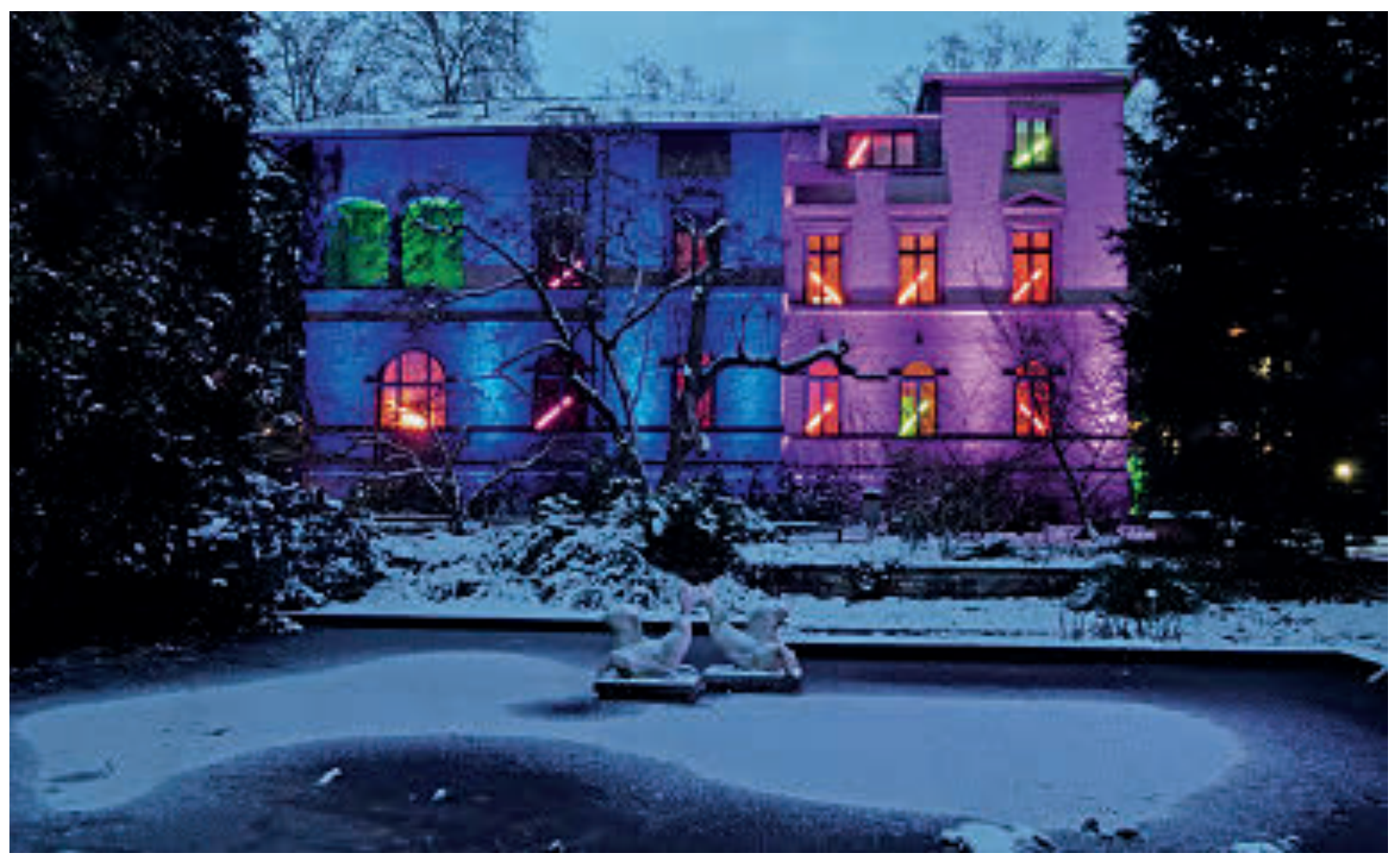

